\title{
Mechanical and Thermal Properties of a Hot-melt Adhesive of Tribrock Copolymer Added with a Miscible Homopolymer
}

\author{
By Hideki KONDO*,† and Akihiko TODA
}

\begin{abstract}
Mechanical and thermal properties of a hot-melt adhesive made from the styrenic triblock copolymer of polystyrene-blockpoly(ethylene-co-propylene)-block-polystyrene, tackifier, and plasticizer oil were examined in terms of the effect of addition of a homopolymer, poly(2,6-dimethyl-1,4-phenylene ether) (PPE). PPE is miscible with the styrene component of the triblock copolymer and has glass transition temperature, $T_{\mathrm{g}}$, higher than that of the styrene component. The properties were examined by shear adhesion failure temperature test, $180^{\circ}$ peel test, dynamic mechanical analysis, and temperature-modulated differential scanning calorimetry. It has been shown that the adhesive properties depend on $T_{\mathrm{g}}$ of the styrene domains, which linearly increases with the content of added PPE. The broader glass transition that resulted from the addition of PPE indicated inhomogeneous distribution of PPE in the styrene domains. Thermal resistance of the adhesive is sustained by physical crosslinks comprising the glassy styrene domains, which are finally broken above $T_{\mathrm{g}}$ of the PPE-rich part of the styrene domains.

KEY WORDS: Hot-melt Adhesive / Triblock Copolymer / Glass Transition /
\end{abstract}

A styrenic A-B-A type triblock copolymer undergoes microphase separation ${ }^{1,2}$ with physical crosslinks formed by styrenic A blocks, which are in the glassy state at room temperature and anchor the flexible rubbery B blocks. If the glass transition temperature, $T_{\mathrm{g}}$, of the rubbery B blocks is sufficiently low, the copolymer can have a broad temperature range with high cohesive strength and low elastic modulus. The copolymer can therefore be used as an adhesive at room temperature, while the loss of physical crosslinks allows thermoplastic processing above the glass transition temperature of the styrene domains, $T_{\mathrm{g}}(\mathrm{StD})$, at about $90^{\circ} \mathrm{C}$. For those reasons, the copolymer can have practical application as a hotmelt adhesive that can be processed at high temperatures, and which recovers its cohesive strength when cooled to ambient temperature. ${ }^{3}$ Hot-melt adhesives have a number of benefits including good peel strength, the ability to bond to rough surfaces, heavier coating weight, and the environmental benefit of not using organic solvents in processing. ${ }^{3}$

The loss of crosslinks above $T_{\mathrm{g}}(\mathrm{StD})$ is an advantage of hotmelt adhesives. However, it also sets an upper limit to the service temperature of the adhesive, which fluidizes and undergoes a sharp drop of cohesive force and elastic modulus above $T_{\mathrm{g}}(\mathrm{StD})$. Because of this thermomechanical property, hot melt adhesive made from styrenic triblock copolymer has lower thermal resistance in comparison with other adhesives made from elastomers with chemical crosslinks.

The key to improving thermal resistance is control of $T_{\mathrm{g}}(\mathrm{StD})$ of the styrene domains. Increase in $T_{\mathrm{g}}(\mathrm{StD})$ can be achieved by mixing with a homopolymer that is miscible with the styrene component and has higher $T_{\mathrm{g}}$, as has been reported. ${ }^{4}$ To understand the limit of the thermal resistance of the adhesive made of styrenic copolymers, we examine, in the present study, the correlation of the thermal resistance of the hot melt adhesive with $T_{\mathrm{g}}(\mathrm{StD})$ of the styrene domains. The adhesive was made from polystyrene-block-poly(ethylene-copropylene)-block-polystyrene (SEPS, a styrenic triblock copolymer), tackifier (TF) and plasticizer oil (OIL), together with a homopolymer, poly(2,6-dimethyl-1,4-phenylene ether) (PPE), which is miscible with the styrene components and has $T_{\mathrm{g}} \sim 165^{\circ} \mathrm{C} .^{5}$ The mechanical and thermal properties were analyzed by shear adhesion failure temperature test, $180^{\circ}$ peel test, dynamic mechanical analysis, and temperature-modulated differential scanning calorimetry.

\section{EXPERIMENTAL}

\section{Materials}

A linear triblock copolymer of SEPS (SEPTON 2007, $M_{\mathrm{w}}=83,000$, triblock contents $=100 \%$, styrene contents $=$ 30 wt \%, Kuraray Co., Ltd) was chosen as the base material in this study. The tackifier (TF) used was a hydrogenated terpene resin (Clearon P-150, Softening Point $=152 \pm 5^{\circ} \mathrm{C}$, Yasuhara Chemical Co., Ltd.), the OIL was paraffinic oil (Diana Process Oil PW-90, kinematic viscosity at $100{ }^{\circ} \mathrm{C}=11.25 \mathrm{~mm}^{2} / \mathrm{s}$, paraffinic carbons $=71.7 \%$, Naphthenic carbons $=28.3 \%$, Aromatic carbons $=0 \%$, and the endblock-associating homopolymer used was PPE (Noryl SA-120, $M_{\mathrm{w}}=6,300, M_{\mathrm{n}}=$ 2,350, $T_{\mathrm{g}}=165^{\circ} \mathrm{C}$, softening point $=210^{\circ} \mathrm{C}$, GE Plastics), together with a phenolic antioxidant (Songnox 1010 made by Sumitomo chemical). Atactic polystyrene (a-PS, Toyo Styrol GP MW-1-301, MFR = 1.9 g/10 min (JISK7210), Vicat softening point $=91^{\circ} \mathrm{C}(50 \mathrm{~N}$ load, JISK7206), TOYO STYRENE Co., Ltd.) was used for additional measurements of mechanical properties.

\footnotetext{
Graduate School of Integrated Arts and Sciences, Hiroshima University, Higashi-Hiroshima 739-8521, Japan

'Present address: Yasuhara Chemical, Co., Ltd., 800-111 Ukaicho, Fuchu 726-0002, Japan

*To whom correspondence should be addressed (Tel: +81-847-40-1503, Fax: +81-847-40-1504, E-mail: kondou@yschem.co.jp).
} 
Table I. Characteristics of the blends

\begin{tabular}{|c|c|c|c|c|c|c|c|}
\hline \multirow{2}{*}{$\begin{array}{c}\text { Sample } \\
\text { name }\end{array}$} & \multirow{2}{*}{$\begin{array}{c}\text { PPE/StD } \\
(w t \%)^{1)}\end{array}$} & \multirow{2}{*}{$\begin{array}{c}\text { StD } \\
(w t \%)^{2)}\end{array}$} & \multirow{2}{*}{$\begin{array}{c}T_{\mathrm{g}}(\mathrm{StD}) \\
\left({ }^{\circ} \mathrm{C}\right)^{3)}\end{array}$} & \multicolumn{4}{|c|}{ Composition ${ }^{4)}$} \\
\hline & & & & $\begin{array}{l}\text { SEPS } \\
\text { (wt \%) }\end{array}$ & $\begin{array}{c}\mathrm{TF} \\
\text { (wt \%) }\end{array}$ & $\begin{array}{c}\text { OIL } \\
(w t \%)\end{array}$ & $\begin{array}{c}\text { PPE } \\
\text { (wt \%) }\end{array}$ \\
\hline MIX1-0\% & 0.0 & 12.0 & 84.9 & 40.0 & 40.0 & 20.0 & 0.0 \\
\hline MIX1-8\% & 7.7 & 12.9 & 88.6 & 39.6 & 39.6 & 19.8 & 1.0 \\
\hline MIX1-29\% & 29.4 & 16.2 & 121.4 & 38.1 & 38.1 & 19.0 & 4.8 \\
\hline MIX1-46\% & 45.5 & 20.0 & 133.0 & 36.4 & 36.4 & 18.2 & 9.1 \\
\hline MIX2-0\% & 0.0 & 21.0 & 86.2 & 70.0 & 20.0 & 10.0 & 0.0 \\
\hline MIX2-8\% & 7.7 & 22.4 & 96.2 & 68.8 & 19.7 & 9.8 & 1.7 \\
\hline MIX2-29\% & 29.4 & 27.4 & 110.6 & 64.4 & 18.4 & 9.2 & 8.0 \\
\hline MIX2-46\% & 45.5 & 32.8 & 123.7 & 59.6 & 17.0 & 8.5 & 14.9 \\
\hline
\end{tabular}

1) Weight fraction of added PPE in the styrene domain in wt \%

2) Weight fraction of the styrene domain in wt \%

3) Grass transition temperature of styrene domains characterized by the inflection points of "Reversing $C_{p}$ " measured by T-MDSC.

4) SEPS: Septon2007, TF: Clearon P150, OIL: Process Oil PW-90, PPE: Noryl SA120

Table II. Characteristics of the blends without TF and OIL

\begin{tabular}{|c|c|c|c|c|c|c|c|}
\hline \multirow{2}{*}{$\begin{array}{c}\text { Sample } \\
\text { name }\end{array}$} & \multirow[b]{2}{*}{$\begin{array}{c}\text { PPE/StD } \\
(w t \%)^{1)}\end{array}$} & \multirow{2}{*}{$\begin{array}{c}\mathrm{StD} \\
(w t \%)^{2)}\end{array}$} & \multirow[b]{2}{*}{$\begin{array}{c}T_{\mathrm{g}}(\mathrm{StD}) \\
\left({ }^{\circ} \mathrm{C}\right)^{3)}\end{array}$} & \multicolumn{4}{|c|}{ Composition ${ }^{4)}$} \\
\hline & & & & $\begin{array}{l}\text { SEPS } \\
\text { (wt \%) }\end{array}$ & $\begin{array}{c}\mathrm{TF} \\
\text { (wt \%) }\end{array}$ & $\begin{array}{c}\text { OIL } \\
\text { (wt \%) }\end{array}$ & $\begin{array}{l}\text { PPE } \\
\text { (wt \%) }\end{array}$ \\
\hline TBC0\% & 0.0 & 30.0 & 92.5 & 100.0 & - & - & 0.0 \\
\hline TBC8\% & 7.7 & 31.7 & 102.6 & 97.6 & - & - & 2.4 \\
\hline TBC29\% & 29.4 & 37.8 & 120.3 & 88.9 & - & - & 11.1 \\
\hline TBC46\% & 45.5 & 44.0 & 130.4 & 80.0 & - & - & 20.0 \\
\hline
\end{tabular}

1) Weight fraction of added PPE in the styrene domain in wt \%

2) Weight fraction of the styrene domain in wt \%

3) Grass transition temperature of styrene domains characterized by the inflection points of "Reversing $C_{p}$ " measured by T-MDSC.

4) SEPS: Septon2007, TF: Clearon P150, OIL: Process Oil PW-90, PPE: Noryl SA120

Preparation of Mixture. The blends of SEPS, TF, and OIL were prepared with addition of PPE. In the blends, TF and OIL are mainly associated with the EP domains of SEPS, and PPE with the styrene domains., 5 The weight fraction of PPE relative to the styrene component, $\mathrm{PPE} / \mathrm{StD}$, was $0.0,7.7,29.4$ or $45.5 \%$, as shown in Table I (MIX1 and MIX2 series). These samples were prepared by mixing the components in a 1-liter double arm kneader under a nitrogen atmosphere. The order of addition of the components to the kneader was as follows. SEPS, PPE and antioxidant were kneaded for $60 \mathrm{~min}$ at $180^{\circ} \mathrm{C}$, then TF was added and kneading continued for $20 \mathrm{~min}$ at $200^{\circ} \mathrm{C}$. Finally, OIL was added and kneading continued for $30 \mathrm{~min}$ at $200^{\circ} \mathrm{C}$.

To examine blends of the copolymer with PPE in the absence of TF and OIL, the samples shown in Table II (TBC series) were prepared by solvent-casting. SEPS and PPE were dissolved in toluene ( $\mathrm{ca}$. $20 \mathrm{wt} \%$ solution) at room temperature. The solvent was evaporated slowly over $7 \mathrm{~d}$ at $30^{\circ} \mathrm{C}$, and the blends were dried under vacuum until no further weight loss was observed (about $3 \mathrm{~d}$ ). The resulting samples were annealed at $170^{\circ} \mathrm{C}$ under vacuum for $4 \mathrm{~h}$.

\section{Methods}

Atomic Force Microscopy (AFM). AFM images were obtained using an SPA-3800-DMF (SII Nano Technology Inc.) instrument in tapping mode, with standard Si probes. Phase contrast images were recorded.
Shear Adhesion Failure Temperature (SAFT) Test. The SAFT test examines the thermal resistance under constant shear at an elevated temperature. Stripe-shaped specimens with average thickness $60 \mu \mathrm{m}$ were prepared by melt coating. The specimens were pressed between two aluminum sheets (bonding area $10 \times 25 \mathrm{~mm}$ ) under applied pressure of $0.2 \mathrm{MPa}$ at $120^{\circ} \mathrm{C}$ for $1 \mathrm{~min}$. The heating rate was $2^{\circ} \mathrm{Ch}^{-1}$ and the load applied to the specimen was $100 \mathrm{gf}$. The SAFT value is defined as the temperature at which bonding failed.

$\mathbf{1 8 0}^{\circ}$ Peel Test. Specimens were prepared by melt coating onto a $38 \mu \mathrm{m}$ thick polyester film; the average thickness of the coating was $40 \mu \mathrm{m}$. The specimen was then pressed onto $100 \mu \mathrm{m}$ thick aluminum sheet, $60 \times 25 \mathrm{~mm}$ in area and cleaned with acetone, using a heat seal tester (TP-701-B, Tester Sangyo) at $0.02 \mathrm{MPa}$ and $120^{\circ} \mathrm{C}$ for $10 \mathrm{~min}$. The composite specimen was cut into $20 \mathrm{~mm}$ wide rectangles and stored at room temperature for at least $24 \mathrm{~h}$. The $180^{\circ}$ peel measurements were carried out using a universal tensile testing machine (TENSILON RTC-100, A\&D Co., Ltd.) at crosshead speed $2.0 \mathrm{~mm} \mathrm{~min}^{-1}$ in the temperature range $90-140^{\circ} \mathrm{C}$, in a temperature-controlled bath.

Dynamic Mechanical Analysis (DMA). Viscoelastic properties (storage modulus and loss tangent, $\tan \delta$ ) of the samples were determined using a rheometer (ARES, TA Instruments) in the $12 \mathrm{~mm}$ parallel plate mode, with temperature sweep from -70 to $250{ }^{\circ} \mathrm{C}$ and frequency sweep from 0.01 to $10 \mathrm{~Hz}$, at constant strain of $0.05 \%$. The gap between the plates was about $1 \mathrm{~mm}$. 
Temperature-Modulated Differential Scanning Calorimetry (T-MDSC). To determine glass transition temperature, T-MDSC was used for the measurement of the "Reversing Heat Flow" with a DSC (Q-100, TA Instruments). The samples were cooled to $-80^{\circ} \mathrm{C}$ and kept at that temperature for $5 \mathrm{~min}$, and then heated at the mean temperature ramp of $1^{\circ} \mathrm{C} \mathrm{min}-1$ with modulation amplitude $\pm 0.2^{\circ} \mathrm{C}$ and period $60 \mathrm{~s}$.

\section{RESULTS AND DISCUSSION}

In this study, blends of triblock copolymer (SEPS), TF and OIL, with added PPE homopolymer were examined in terms of their performance as hot-melt adhesives at high temperatures. PPE is compatible with the styrene component of the blend and thus supposed to change the glass transition temperature of that component. The samples were tested as described below to investigate the effects of PPE on mechanical and thermal properties.

\section{AFM}

The AFM images in Figure 1 show the microphaseseparated morphology of the blends of MIX1-0\% to $46 \%$. The dark areas correspond to the styrene domains; the other areas are the EP domains. The weight fraction of styrene domain in the blend, $\mathrm{StD}$, increases with the amount of added PPE because of the compatibility of PPE with the styrene components, so that the morphology undergoes successive change from micelles (MIX1-0\%, 8\% and 29\%) to cylinders (MIX1-46\%) with increasing amount of PPE.
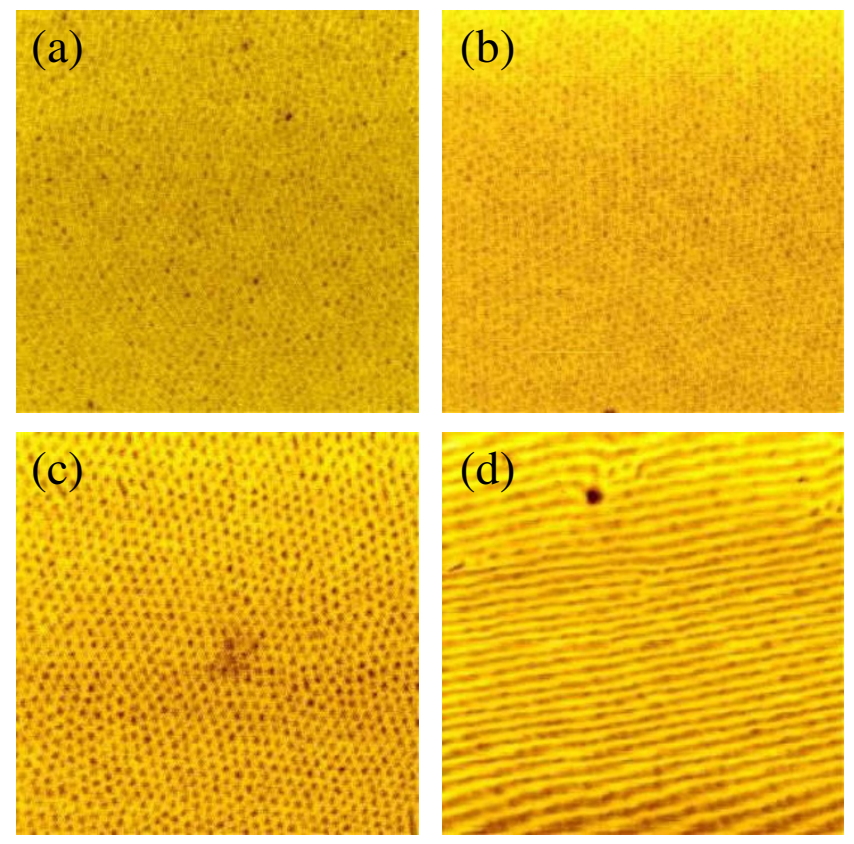

Figure 1. $1 \times 1 \mu \mathrm{m}^{2}$ phase contrast images of tapping mode AFM of the mixtures, (a) MIX1-0\%, (b) MIX1-8\%, (c) MIX1-29\% and (d) MIX1$46 \%$.

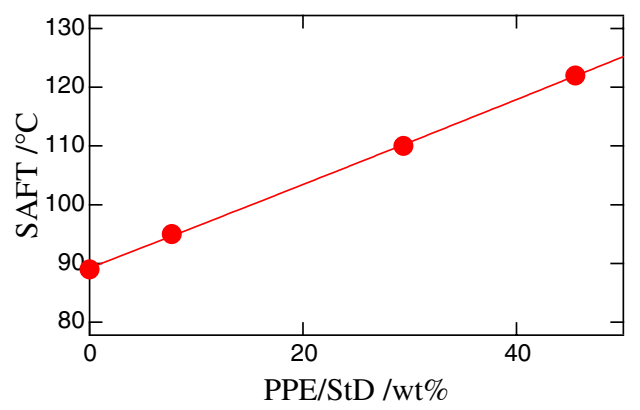

Figure 2. SAFT as a function of PPE/StD for the adhesives of MIX1 series.

\section{SAFT Test}

SAFT is the measure of thermal resistance under shear at a constant rate of increase of temperature. ${ }^{7}$ The SAFT value is defined as the temperature at which bonding fails. All samples showed cohesive failure, so that adhesive remained on both substrates. The controlling parameters of the SAFT test are the heating rate and the applied load. Heating rate, in particular, significantly influences the SAFT value due to the time lag of bonding failure after the onset of deformation. The test was carried out at $2{ }^{\circ} \mathrm{Ch}^{-1}$ heating rate, which was sufficiently small to minimize this effect. Figure 2 shows that the SAFT value increased linearly with addition of PPE, i.e., with PPE/ $\mathrm{StD}$. The slope of the best fit line was $0.72{ }^{\circ} \mathrm{C}(\mathrm{wt} \%)^{-1}$ and the intercept was $88.8^{\circ} \mathrm{C}$. The linear relationship suggests that addition of PPE increases the glass transition temperature of the endblock styrene domain, $T_{\mathrm{g}}(\mathrm{StD})$, and consequently increases the thermal resistance of the adhesives at higher temperatures.

\section{$180^{\circ}$ Peel Test}

For examination of the peeling behavior of adhesives, the controlling parameters are peel speed and the ambient temperature. If the principle of time-temperature superposition ${ }^{3,8}$ is satisfied in terms of the thermomechanical properties of the material, the data obtained for limited ranges of temperature and peel speed can be superimposed to give a master curve of peel strength plotted against peel speed multiplied by a shift factor. Such attempts have been made for several polymer adhesives. ${ }^{9}$ In those cases, the master curves clearly showed a transition from cohesive failure at slower speed (higher temperature) to adhesive failure at faster speed (lower temperature), and a further transition at still faster speed (still lower temperature) from adhesive failure to loss of adhesive strength. Based on those results, it is believed that the performance of adhesives is determined by the viscoelastic properties of the materials.

In relation to the present material, i.e., the blends of triblock copolymer, it is known that the principle of time-temperature superposition is not satisfied due to differing thermomechanical properties, e.g., different glass transition temperatures, of the components, ${ }^{9,10}$ as discussed below in the section on DMA. Consequently, to analyze adhesive performance, it is necessary to examine either broad ranges of peel speed at constant 


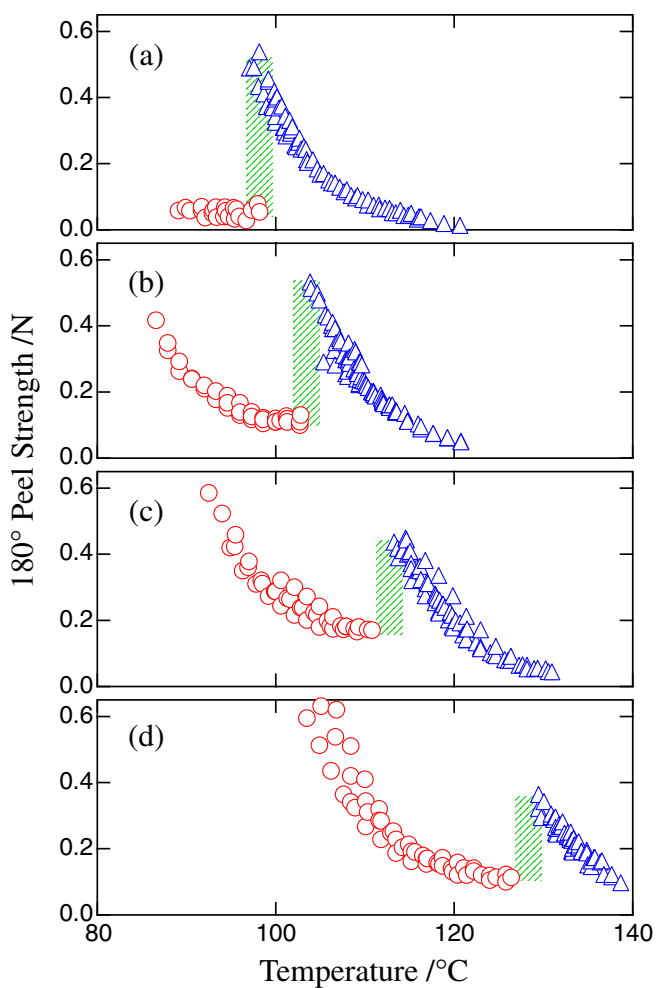

Figure 3. $180^{\circ}$ peel strength of the adhesives, (a) MIX1-0\%, (b) MIX1-8\%, (c) MIX1-29\% and (d) MIX1-46\% as a function of temperature. The symbol of open circle $(\bigcirc)$ denotes the adhesion failure and the open triangle $(\triangle)$ denotes the cohesion failure. The hatched area represents the coexistence region.

temperature, or broad ranges of temperature at fixed peel speed. Because the range of peel speeds is limited by the test instrument, we examined peeling in a wide temperature range with the peel speed fixed at $2 \mathrm{~mm} \mathrm{~min}^{-1}$.

Figure 3 shows the results of the $180^{\circ}$ peel test and the following characteristics (Figure 4) can be seen. With increasing temperature from the low temperature side, the peel strength continuously decreases in adhesive failure mode. At a certain temperature, the peeling mode undergoes a transition from adhesive failure to cohesive failure, followed by the recovery of peel strength. In a narrow temperature range, adhesive failure and cohesive failure coexist, and peeling is preceded by the changeover between those two modes of failure in the manner of stick-slip. With further increase in temperature, the peel strength again decreases in cohesive failure mode. The same behavior was found for all samples examined (see Figure 3).

It is well known that decrease in peel strength with increasing temperature (or decreasing peel speed) and a transition between cohesive failure and adhesive failure are common behaviors in peeling of adhesives. ${ }^{6,12,13}$ The behavior is thought to be due to the change in the viscoelastic properties of the adhesives with temperature and peel speed. That means that the decrease in peel strength with increasing temperature should be due to the decrease in the dynamic elastic modulus with increasing temperature (or slower peel speed). Cohesive failure implies breakdown of stringy adhesives at the peel

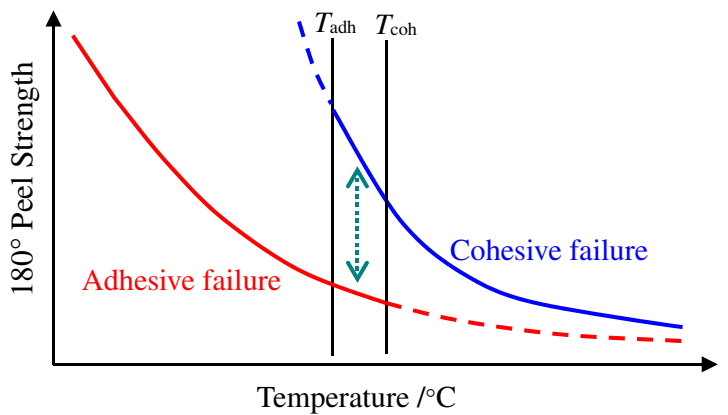

Figure 4. Schematic diagram of the $180^{\circ}$ peel strength as a function of temperature.

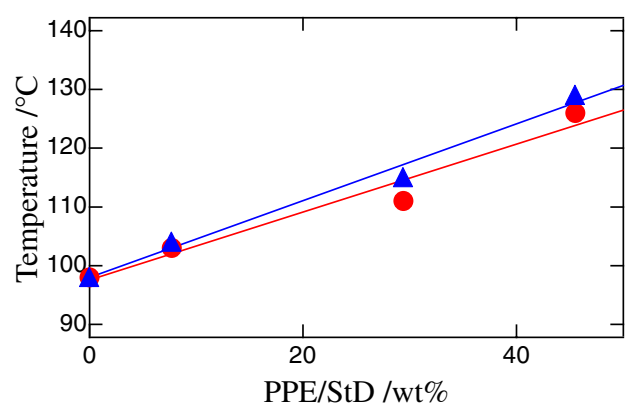

Figure 5. $T_{\text {adh }}(O)$ and $T_{\text {coh }}(\mathbf{\Delta})$ of $180^{\circ}$ peel test in Figure 3 as a function of PPE/StD.

front. The required deformation for breakdown will become easier with decrease in the dynamic elastic modulus at higher temperatures. On the other hand, for adhesive failure at the interface, to explain similar behavior with temperature and peel speed, the adhesive force must be a decreasing function of temperature (increasing function of peel speed), ${ }^{12,13}$ and may also be determined by the viscoelastic properties at the interface. In relation to the transition from adhesive failure to cohesive failure, the transition with increasing temperature indicates that cohesive strength decreases to a greater extent than does the adhesive strength, with increasing temperature (or slower speed).

Figure 5 shows plots of the upper temperature limit of adhesive failure, $T_{\mathrm{adh}}$, and the lower temperature limit of cohesive failure, $T_{\text {coh }}$, as functions of PPE/StD. Both temperatures increase linearly with PPE/StD, as does the SAFT value. The slopes of the best fit lines are 0.60 and $0.66{ }^{\circ} \mathrm{C}(\mathrm{wt} \%)^{-1}$ and the intercepts are 97.6 and $97.1^{\circ} \mathrm{C}$, for $T_{\text {adh }}$ and $T_{\text {coh }}$, respectively. The values obtained for the slope are close to that of the SAFT value. Consequently, the results of both the SAFT test and the $180^{\circ}$ peel test clearly suggest that the thermal resistance of the adhesive performance is strongly influenced by change of PPE/StD, which is presumed to cause a shift of $T_{\mathrm{g}}(\mathrm{StD})$. In the DMA and T-M DSC measurements discussed below, we examine the correlation between $T_{\mathrm{g}}(\mathrm{StD})$ and the thermomechanical properties of the adhesives.

\section{DMA}

DMA examines the variation in viscoelastic properties of 


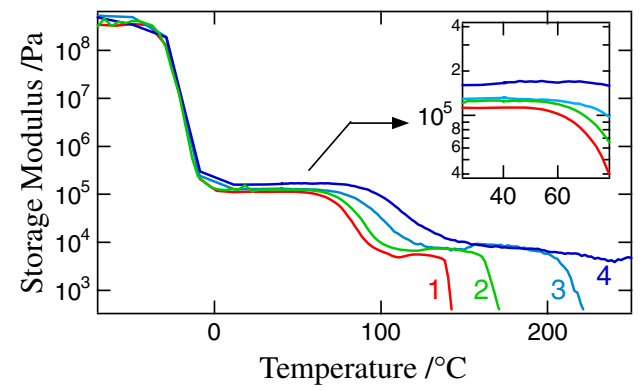

Figure 6. Storage modulus of DMA of the adhesives, (1) MIX $1-0 \%$, (2) MIX1-8\%, (3) MIX1-29\% and (4) MIX1-46\% as a function of temperature at the frequency of $0.01 \mathrm{~Hz}$.

the materials with temperature and applied frequency, via the change in the dynamic complex elastic modulus. ${ }^{8}$ Figure 6 shows the change in modulus with temperature at a fixed frequency of $0.01 \mathrm{~Hz}$. With increasing temperature, stepwise decreases in the real part of the dynamic elastic modulus, i.e., the storage modulus, $G^{\prime}$, show successive changes in the viscoelastic properties of the adhesives. Those changes are identified with the glass transition of the EP component, $T_{\mathrm{g}}(\mathrm{EPD})$, at low temperature $\left(-20^{\circ} \mathrm{C}\right)$, the glass transition of the styrene component, $T_{\mathrm{g}}(\mathrm{StD})$ at higher temperature $(80-$ $130^{\circ} \mathrm{C}$ ), and the order-disorder transition of copolymer, $T_{\mathrm{ODT}}$, at still higher temperature (above $140^{\circ} \mathrm{C}$ ). $T_{\mathrm{g}}(\mathrm{EPD})$ remained unchanged for all samples with different PPE content, confirming that added PPE was incompatible with the EP component.

In the present study, we are concerned with the shift in the glass transition temperature of the styrene component with the addition of PPE, which is thought to be compatible with the styrene component. Above the glass transition, the crosslinking points disappear from the adhesives and the thermal resistance is lost. It is clear from the temperature dependence of the storage modulus (Figure 6) that the stepwise decrease corresponding to $T_{\mathrm{g}}(\mathrm{StD})$ shifted from 80 to $130^{\circ} \mathrm{C}$ with increasing $\mathrm{PPE} / \mathrm{StD}$. Since the glass transition temperature of PPE is known to be $165^{\circ} \mathrm{C}$, the temperature shift of the stepwise decrease of $G^{\prime}$ suggests that the added PPE is compatible with the styrene endblock components, as has been reported. ${ }^{14}$

Figure 7 shows the temperature dependence of $\tan \delta$ between 50 and $150{ }^{\circ} \mathrm{C}$. We have defined $T_{\mathrm{g}}(\mathrm{StD})$ as the peak temperature of $\tan \delta$. Figure 8 shows that the peak temperature increases linearly with PPE/StD: the slope of the best fit line is $0.66^{\circ} \mathrm{C}$ (wt \%) $)^{-1}$ and the intercept is $89.3^{\circ} \mathrm{C}$. The slope is in good agreement with the slopes determined for thermal resistance by the SAFT and $180^{\circ}$ peel tests.

There are two notable aspects of the change in the modulus with addition of PPE. First, the absolute value of the storage modulus at the plateau between $T_{\mathrm{g}}(\mathrm{EPD})$ and $T_{\mathrm{g}}(\mathrm{StD})$ increases with increasing PPE/StD. It seems that the increase is caused by the volume fraction of glassy styrene domain, and by the change in morphology from micelle to cylinder. Second, in relation to the order-disorder transition of the microphaseseparated structure of the blend, the sharp drop in the storage

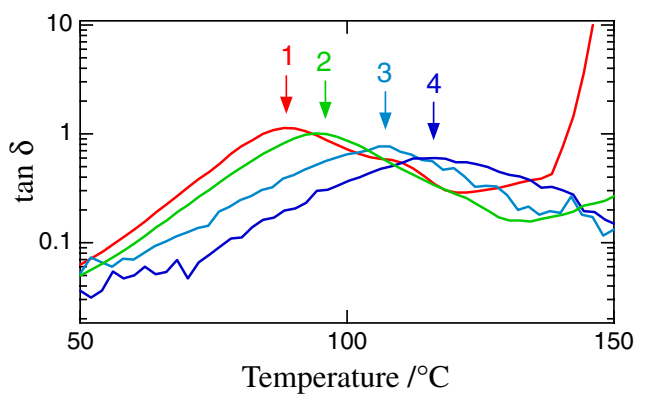

Figure 7. Tan $\delta$ of DMA of the adhesives, (1) MIX1-0\%, (2) MIX1-8\%, (3) MIX1-29\% and (4) MIX1-46\% as a function of temperature at the frequency of $0.01 \mathrm{~Hz}$. Arrows indicates $T_{\mathrm{g}}(\mathrm{StD})$.

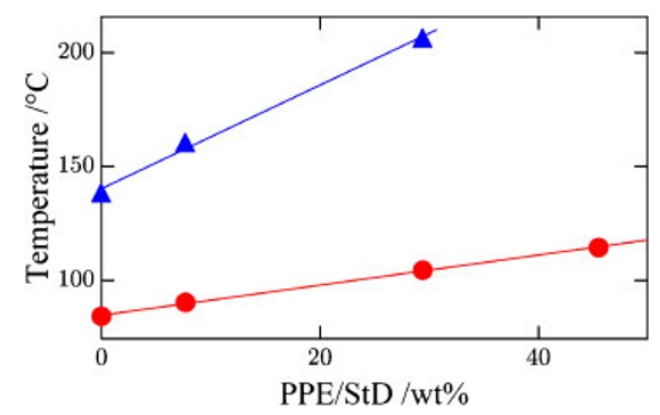

Figure 8. $T_{\mathrm{g}}(\mathrm{StD})(\mathrm{O})$ and $T_{\mathrm{ODT}}(\mathbf{\Delta})$ determined by DMA as a function of PPE/StD for the adhesives of MIX1 series.

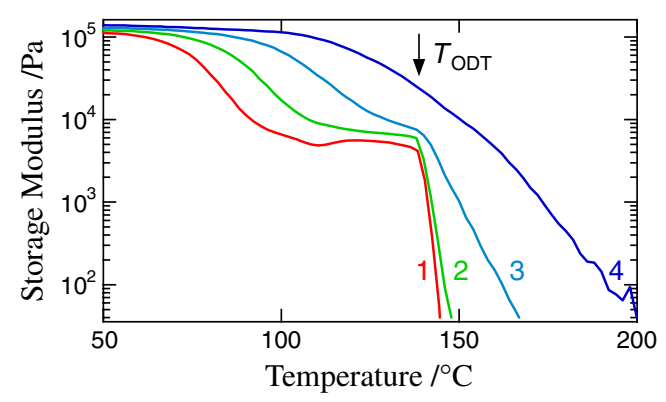

Figure 9. Storage modulus as a function of temperature for the mixture of MIX $1-0 \%$ at the frequency of (1) 0.01 , (2) 0.1 , (3) 1.0 and (4) $10.0 \mathrm{~Hz}$.

modulus at around 140, 160 and $200{ }^{\circ} \mathrm{C}$ for MIX1-0\%, $8 \%$ and $29 \%$, respectively, did not change with applied frequency from 0.01 to $10.0 \mathrm{~Hz}$. A typical case, for MIX1-0\%, is shown in Figure 9. The insensitivity to frequency is strong supporting evidence for the assignment of this sharp drop to a first-order phase transition corresponding to an order-disorder transition. The transition temperature, $T_{\mathrm{ODT}}$, plotted in Figure 8 increases with increasing PPE/StD, so that addition of PPE has a strong influence on $T_{\mathrm{ODT}}$. The slope of $T_{\mathrm{ODT}}$ is $2.27^{\circ} \mathrm{C}$ (wt $\left.\%\right)^{-1}$ and the intercept is $140.3{ }^{\circ} \mathrm{C}$. There is a large difference in the slope of $T_{\mathrm{ODT}}$ and those of SAFT, $T_{\mathrm{adh}}, T_{\mathrm{coh}}$, and $T_{\mathrm{g}}(\mathrm{StD})$ shown in Figures 2, 5 and 8 . It seems, therefore, that the thermal resistance of the adhesive is determined mainly by the viscoelastic property represented by $T_{\mathrm{g}}(\mathrm{StD})$, and not by $T_{\text {ODT }}$ of the triblock copolymer. 


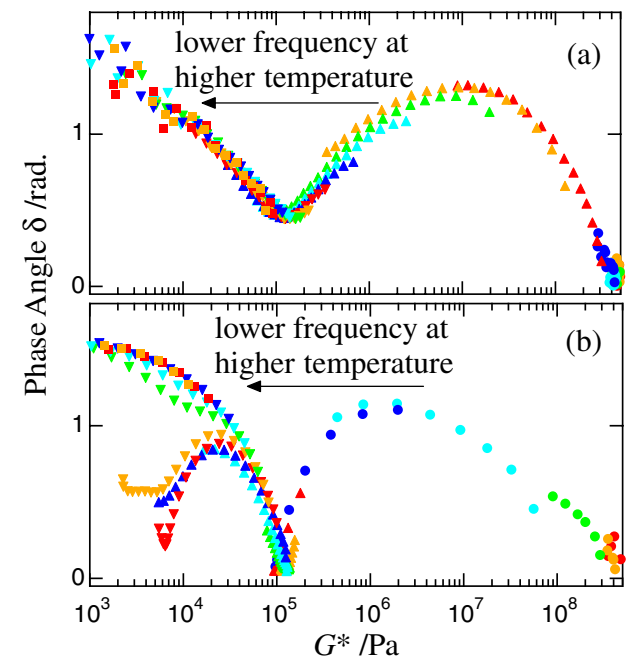

Figure 10. Phase angle, $\delta$, as a function of the dynamic modulus, $G^{*}$, of (a) a-PS and (b) MIX1-0\%. Symbols with the same color and shape denote the measurements at a constant temperature with frequencies of $0.01-10 \mathrm{~Hz}$ : (a) $40-200^{\circ} \mathrm{C}$ at every $10^{\circ} \mathrm{C}$ and (b) $-70-130^{\circ} \mathrm{C}$ at every $20^{\circ} \mathrm{C}$ and $140-190^{\circ} \mathrm{C}$ at every $10^{\circ} \mathrm{C}$.

Figure 10 shows plots of the phase angle, $\delta=\operatorname{atan}\left(G^{\prime \prime} / G^{\prime}\right)$, against the dynamic modulus, $G^{*}=\left(G^{\prime 2}+G^{\prime \prime 2}\right)^{1 / 2}$, obtained from measurements at various temperatures and frequencies for pure a-PS and for the blends of triblock copolymer of MIX1$0 \%$. This kind of plot is utilized to judge the applicability of the time-temperature superposition principle. ${ }^{11}$ The principle is applicable if the data points lie on a single master curve. Figure 10a shows that the data for pure polystyrene lie on a single master curve, but that is not the case for the triblock copolymer blends (Figure 10b). The data points for $G^{*}<10^{5}$ $\mathrm{Pa}$ in Figure $10 \mathrm{~b}$ cannot be represented by a single master curve, and that region corresponds to the glass transition of styrene domains in the ordered state above the order-disorder transition, which occurs for $G^{*}<10^{4} \mathrm{~Pa}$ (see Figure 9). The results confirm that the time-temperature superposition principle is not applicable to the present system due to the inhomogeneous nature of the microphase-separated material. Hence the thermomechanical properties of the material must be analyzed without recourse to time-temperature superposition.

\section{T-MDSC}

For the purpose of determination of $T_{\mathrm{g}}(\mathrm{StD})$, DMA is an indirect method because the transition is associated with a stepwise decrease in the storage modulus of the whole sample. The stepwise decrease in $G^{\prime}$ is caused by the breakdown of the crosslinking points composed of styrene domains that are softening with heating, due to the glass transition. By contrast, thermal measurement by DSC detects directly the change in heat capacity of the sample, and that of the styrene domains.

Due to the small volume fraction of the styrene domains in the adhesive, it is not an easy task to detect the small change in heat capacity associated with the glass transition of those domains. With conventional DSC in which the sample is heated at a constant rate, determination of $T_{\mathrm{g}}$ is made more difficult by inevitable baseline drift. T-MDSC is a relatively

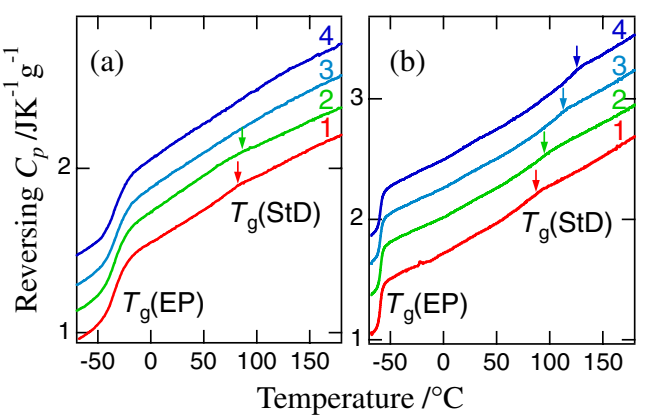

Figure 11. "Reversing $C_{p}$ " of T-MDSC as a function of temperature for (1) MIX1-0\%, (2) MIX1-8\%, (3) MIX1-29\% and (4) MIX1-46\% in (a) and for (1) TBC0\%, (2) TBC8\%, (3) TBC29\% and (4) TBC46\% in (b). The data points are shifted vertically for viewing.

new technique by means of which a periodic modulation in temperature is applied to the sample, and the resultant heat flow response is analyzed. ${ }^{15-17}$ A dynamic complex heat capacity can be determined from the ratio of the modulation amplitudes and the difference in phase angles for the applied modulation frequency. At the glass transition, the real part of the dynamic heat capacity shows a stepwise change, as in the case of the heat capacity obtained by conventional DSC, and the imaginary part shows a peak. ${ }^{18}$ If the modulation period is sufficiently small, the analysis method of T-M DSC is not influenced by baseline drift because only the modulation components are utilized for the analysis. Consequently, the method is frequently applied to detection of the glass transition in polymeric materials.

Figure 11a shows the magnitude of the dynamic heat capacity (referred to as "Reversing $C_{p}$ ") for MIX1-0\% to $46 \%$. Because the proportion of StD was only $12-22 \%$, it was difficult to detect the stepwise change in the "Reversing $C_{p}$ " for the MIX1 series of blends, especially for higher PPE contents. For that reason the TBC series of blends with PPE only (i.e., without TF and OIL) was prepared to enable determination of the glass transition temperature in blends with increased proportion of StD.

Figure $11 \mathrm{~b}$ shows the "Reversing $C_{p}$ " of TBC- $0 \%$ to $46 \%$ blends. The proportions of StD in the TBC series of blends were $30-44 \%$ larger than those in the MIX1 series, so that the change in heat capacity at $T_{\mathrm{g}}(\mathrm{StD})$ was clearly discernable for the TBC series. It is noteworthy that $T_{\mathrm{g}}(\mathrm{EPD})$ is about $-35^{\circ} \mathrm{C}$ in Figure $11 \mathrm{a}$ and $-60^{\circ} \mathrm{C}$ in $11 \mathrm{~b} . T_{\mathrm{g}}(\mathrm{EPD})$ remains unchanged with different PPE contents in both Figure 11a and $11 \mathrm{~b}$, and shifts to lower temperature in the absence of TF and OIL. These results re-confirm that the added PPE is incompatible, and TF and OIL are compatible with the EP component.

The systematic change in $T_{\mathrm{g}}(\mathrm{StD})$ with addition of PPE (Figure 11) can be due to the change in the composition of styrene domains, as is supposed. In addition, because it is known that $T_{\mathrm{g}}$ is dependent on system size, when the size becomes smaller than $c a .100 \mathrm{~nm},{ }^{19}$ the change in $T_{\mathrm{g}}(\mathrm{StD})$ may arise from its dependence on the size of the styrene domains. To differentiate those two effects, we have compared $T_{\mathrm{g}}(\mathrm{StD})$ for the three series of samples that have different proportions of 


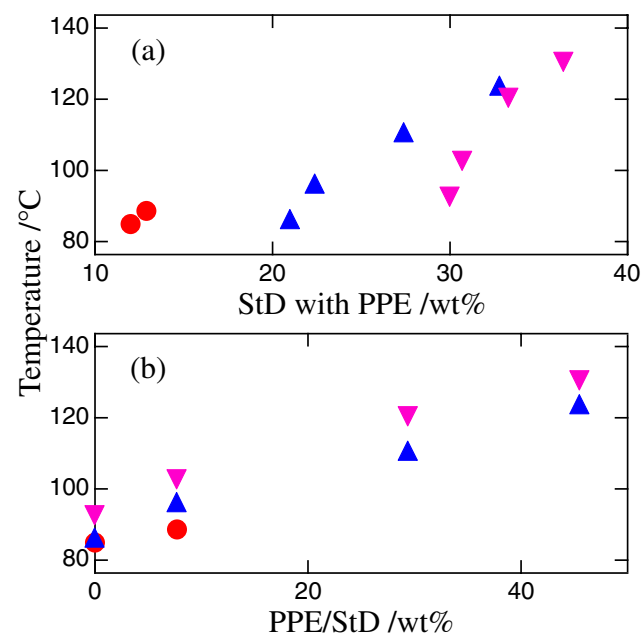

Figure 12. $T_{\mathrm{g}}(\mathrm{StD})$ measured by $\mathrm{T}-\mathrm{MDSC}$ as a function of (a) StD and (b) PPE/StD. Symbols denote MIX1 (O), MIX2 ( $\mathbf{\Delta})$ and TBC $(\boldsymbol{\nabla})$.

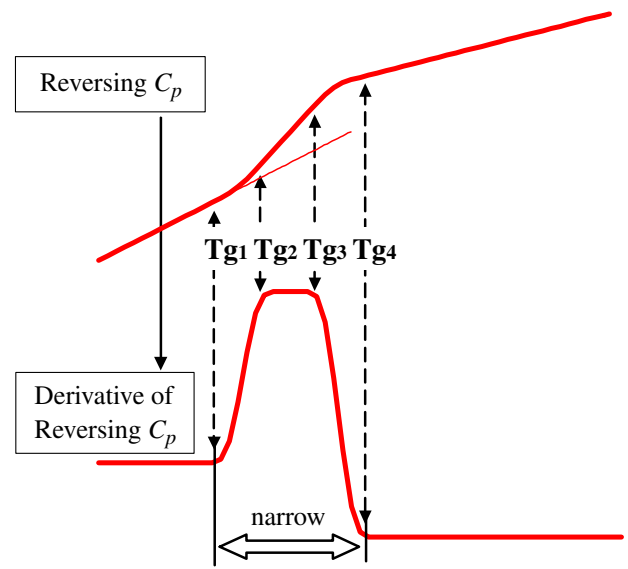

(a)

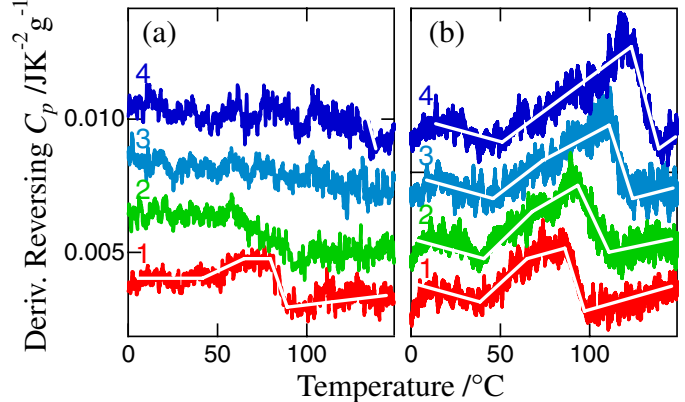

Figure 14. Derivative of the "Reversing $C_{p}$ " shown in Figure 12 as a function of temperature for (1) MIX1-0\%, (2) MIX1-8\%, (3) MIX1-29\% and (4) MIX1-46\% in (a) and for (1) TBC0\%, (2) TBC8\%, (3) TBC29\% and (4) TBC46\% in (b). The data points are shifted vertically for viewing.

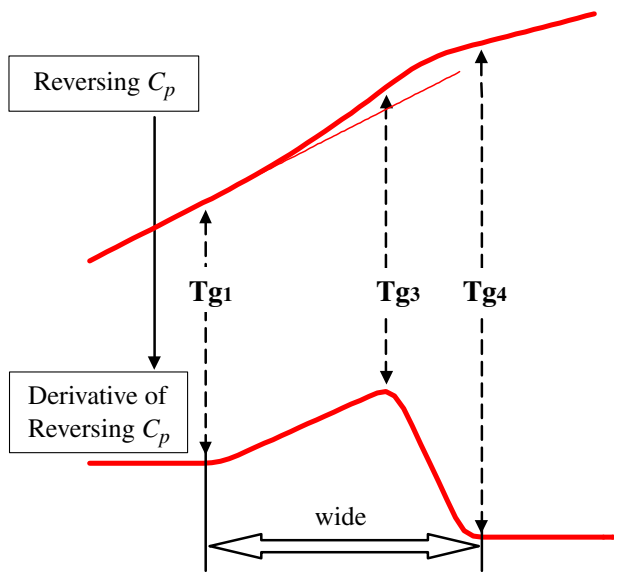

(b)

Figure 13. Schematic diagram of the "Reversing $C_{p}$ " and its derivative in the glass transition for the cases of (a) narrow and (b) broad transition regions. In (b), the slope change, $T_{\mathrm{g} 2}$, is not clearly discernable.

StD, namely MIX1, MIX2 and TBC. Figure 12 shows $T_{\mathrm{g}}(\mathrm{StD})$ as a function of StD with PPE in (a), and as a function of PPE/ $\mathrm{StD}$ in (b). If $T_{\mathrm{g}}(\mathrm{StD})$ is determined only by the system size of styrene domains, which is characterized by StD, the data in Figure 12a should lie on a single master curve, which is clearly not the case. On the other hand, Figure $12 \mathrm{~b}$ shows that the change in $T_{\mathrm{g}}(\mathrm{StD})$ is determined mainly by the change in the composition, expressed by PPE/StD, of styrene domains with addition of PPE.

In terms of $T_{\mathrm{g}}(\mathrm{StD})$, the change in the heat capacity with composition has been quantitatively analyzed in the following manner. As schematically shown in Figure 13a, the glass transition has a stepwise change of the "Reversing $C_{p}$ " with a certain width of the transition region. In order to emphasize the change, the temperature derivative of the "Reversing $C_{p}$ " has been calculated. The resultant curve of the derivative is trapezoidal in shape, as shown in Figure 13a. The glass transition can be characterized by the inflection points, $T_{\mathrm{g} 1}, T_{\mathrm{g} 2}$, $T_{\mathrm{g} 3}$ and $T_{\mathrm{g} 4}$.
Figure 14b shows the derivative of the "Reversing $C_{p}$ " of the TBC series shown in Figure $11 \mathrm{~b}$ as a function of temperature. The trapezoidal shape of the curves is clearly confirmed in $\mathrm{TBC} 0 \%$ and $\mathrm{TBC} 8 \%$, while the change is not as clear for $\mathrm{TBC} 29 \%$ and $\mathrm{TBC} 46 \%$. As indicated in Figure 13b, for higher content of PPE/StD, the width of the transition region increases, so that the height of the trapezoid becomes smaller. As a result the inflection point of the derivative becomes unclear, as shown in Figure 13b. The broadening of the transition region for higher PPE content suggests increasing inhomogeneity of PPE distribution in the styrene domains. Figure 14a shows similar plots of the derivative of the "Reversing $C_{p}$ " calculated for the adhesives of the MIX1 series (Figure 11a). A small change recognized as a trapezoidal shape can be seen for MIX1-0\%, but not for the other members of the series. It seems that the broadening of the transition region for higher fractions of PPE prevented confirmation of the change in heat capacity for the adhesives with smaller relative ratio of styrene domains. 


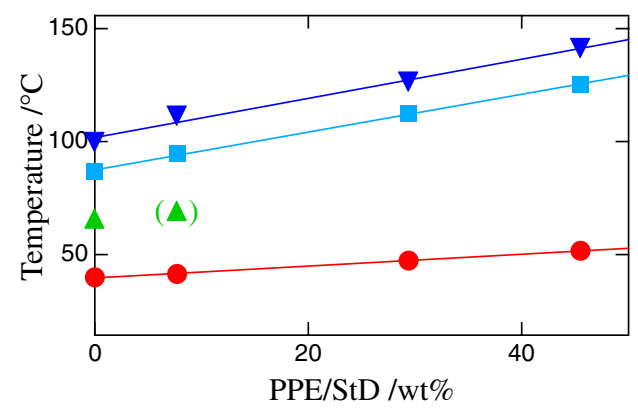

Figure 15. $T_{\mathrm{g}}(\mathrm{StD})$ determined from T-MDSC results in Figure $14 \mathrm{~b}$ for TBC series as a function of PPE/StD: $T_{\mathrm{g} 1}(\mathbf{O}), T_{\mathrm{g} 2}(\mathbf{\Delta}), T_{\mathrm{g} 3}(\square)$ and $T_{\mathrm{g} 4}(\boldsymbol{\nabla})$.

Figure 15 shows the inflection points $T_{\mathrm{g} 1}, T_{\mathrm{g} 2}, T_{\mathrm{g} 3}$ and $T_{\mathrm{g} 4}$ as a function of PPE/StD for the TBC series. $T_{\mathrm{g} 3}$ and $T_{\mathrm{g} 4}$ increase linearly with PPE/StD, while $T_{\mathrm{g} 2}$ could not be evaluated for higher PPE content. The slope of the onset temperature of the glass transition, $T_{\mathrm{g} 1}$, is much smaller than the slopes of the other transition temperatures. As noted above, the difference suggests broadening of the glass transition due to inhomogeneous distribution of PPE in the styrene domains. Inhomogeneous distributions have been reported for blends of the triblock copolymer with homopolymers, from analyses by DMA $^{14}$ and conventional DSC. ${ }^{20,21}$ It has been supposed that the homopolymers are dissolved in the styrene domains with lower concentration near the interface and higher concentration in the core. The present study provides support for that supposition from analysis of the change in the slopes of the inflection points. It seems that $T_{\mathrm{g} 1}$ corresponds to the transition temperature of pure styrene and $T_{\mathrm{g} 3}$ and $T_{\mathrm{g} 4}$ correspond to PPErich styrene components.

It should be taken into consideration that the broad glass transition may be simply due to inhomogeneity of mixing. To ascertain the effect of mixing, we prepared the MIX1-0\% and MIX2-8\% blends by two different methods, namely kneading the molten blend, and dissolving the blend in toluene. There were negligible differences in the results obtained with those blends prepared by the two different methods of mixing. For this reason, we have concluded that the broad transition must be due to inhomogeneous distribution of PPE in the styrene domains.

\section{Comparison of the Results}

Figure 16 shows the changes in the characteristic temperatures as a function of PPE/StD. SAFT represents the thermal resistance of the adhesive under shear, and $T_{\text {adh }}$ and $T_{\text {coh }}$ are the temperatures associated with the change from adhesive failure to cohesive failure in the $180^{\circ}$ peel test. Above those temperatures, the adhesive remains on both substrates in cohesive failure, which causes serious problem in adhesion performance. Consequently, $T_{\text {adh }}$ and $T_{\text {coh }}$ are important measures of adhesive performance at high temperature.

In the comparison of the slopes from the mechanical analysis of the adhesive performance, the SAFT test and the

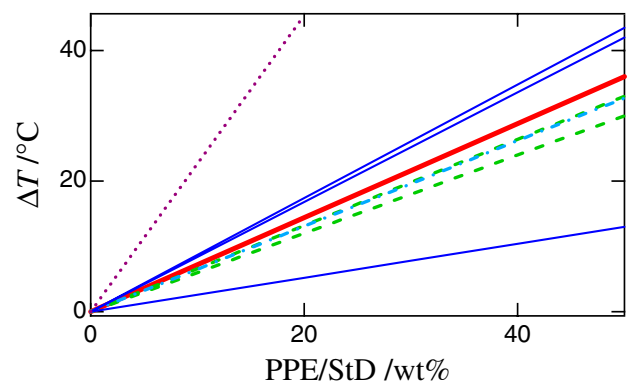

Figure 16. Slopes of the shift in the transition temperatures as a function of PPE/StD determined by SAFT test (thick line), $180^{\circ}$ peel test ( $\left.T_{\text {adn }}, T_{\text {coh }}\right)$ (broken lines), $T_{\text {g }}(\mathrm{StD})$ (alternate long and short dash line) and $T_{\mathrm{ODT}}$ (dotted line) by DMA and $T_{\mathrm{g}}(\mathrm{StD})\left(T_{\mathrm{g} 1}, T_{\mathrm{g} 3}, T_{\mathrm{g} 4}\right)$ (thin lines) by T-MDSC.

$180^{\circ}$ peel test ( $T_{\text {adh }}$ and $\left.T_{\text {coh }}\right)$, and from the thermal analysis by T-MDSC (giving $T_{\mathrm{g} 1}, T_{\mathrm{g} 3}$ and $T_{\mathrm{g} 4}$ ), the slopes of $T_{\mathrm{g} 3}$ and $T_{\mathrm{g} 4}$ from T-MDSC were close to those obtained from the mechanical measurements. The implication is that the performance of the hot-melt adhesive is determined by the glass transition temperature of the PPE-rich part of the styrene domains. For polymer adhesives without physical crosslinks, it has been suggested that the transition from adhesive failure to cohesive failure is caused by the change in the viscoelastic properties of the adhesive from the rubber plateau to the flow region. In the present system with physical crosslinks of the styrene domains, softening of the PPE-rich part of the styrene domains introduces a large reduction in the dynamic elastic modulus, which occurs at $T_{\mathrm{g}}(\mathrm{StD})$ of DMA showing a change similar to $T_{\mathrm{g} 3}$ and $T_{\mathrm{g} 4}$, as seen in Figure 16. It follows that for the adhesive property, softening of the physical crosslinking points is the determining factor, corresponding to the change from rubber plateau to flow region in adhesives without physical crosslinks.

\section{CONCLUSION}

We have examined the thermomechanical properties of a hot-melt adhesive made from blends of SEPS, TF and OIL, with the addition of PPE, which is compatible with the styrene components. Adhesives with varying PPE content have been examined using the SAFT test, $180^{\circ}$ peel test, DMA and TMDSC. The SAFT test and $180^{\circ}$ peel test provide information on the thermal resistance of the adhesive performance, DMA the viscoelastic properties, and T-MDSC the thermal properties. Using DMA and T-MDSC, it is confirmed that $T_{\mathrm{g}}(\mathrm{StD})$ and $T_{\mathrm{ODT}}$ are strongly influenced by the addition of PPE, while $T_{\mathrm{g}}(\mathrm{EPD})$ is not. The results show that PPE is mainly compatible with the styrene component and incompatible with the EP component of the blends. ${ }^{14}$ By examining the change in heat capacity, it has also been shown that the temperature range of the glass transition becomes broader with the addition of PPE. The broadening suggests inhomogeneous distribution of PPE in the styrene domains; ${ }^{20}$ similar behavior has been reported for blends of a styrene-butadiene-styrene triblock copolymer with homopolymers. ${ }^{21}$ 
The thermal resistance of adhesive performance has been examined by the SAFT test and $180^{\circ}$ peel test in a wide temperature range at a fixed speed of deformation. The measurements in a wide temperature range were necessary because the time-temperature superposition principle is not applicable to blends of copolymers with different viscoelastic properties. The SAFT result is in accordance with the change in the glass transition temperature of the styrene component. The $180^{\circ}$ peel test clearly suggests that the transition of the peel mode is dependent on the glass transition temperature of the styrene component. The results of the SAFT test and $180^{\circ}$ peel test indicate that the upper temperature limit of the glass transition determines the adhesive performance at high temperature. That conclusion is reasonable because the mechanical properties of the system undergo drastic change above $T_{\mathrm{g}}(\mathrm{StD})$, due to the loss of crosslinking points sustained by the PPE-rich part of the styrene component, which has the highest glass transition temperature due to the higher PPE content.

Acknowledgment. The authors are grateful to Profs. K. Taguchi and S. Tanaka of Hiroshima University for helpful discussions. This work was partially supported by MEXT Japan, Grant-in-Aid for Scientific Research on Priority Areas, "Creation of Non-equilibrium Soft Matter Physics."

Received: July 17, 2008

Accepted: October 7, 2008 Published: November 19, 2008

\section{REFERENCES}

1. K. Binder, Adv. Polym. Sci., 112, 181 (1994).

2. V. Castelletto and I. W. Hamley, Curr. Opin. Solid State Mater. Sci., 8, 426 (2004).

3. A. V. Pocius, "Adhesion and Adhesives Technology-an Introduction," Hanser, Munich, 2002, Chap. 5 and 10.

4. T. A. Huy, R. Adhikari, T. H. Lupke, G. H. Michler, and K. Knoll, Polym. Eng. Sci., 44, 1534 (2004).

5. C. Mazard, L. Benyahia, and J. F. Tassin, Polym. Int., 52, 514 (2003).

6. D. Satas, "Handbook of Pressure Sensitive Adhesive Technology, 2nd ed.," Van Nostrand Reinhold, New York, 1989, Chap. 5 and 13.

7. N. Nakajima, R. Babrowicz, and E. R. Harrell, J. Appl. Polym. Sci., 44, 1437 (1992).

8. J. D. Ferry, "Viscoelastic properties of Polymers," Wiley, New York, 1980 .

9. a) M. Mitoh and K. Nakao, J. Adhesion Soc. Jpn., 19, 433 (1983). b) M. Mitoh and K. Nakao, J. Adhesion Soc. Jpn., 19, 485 (1983). c) M. Mitoh and K. Nakao, J. Adhesion Soc. Jpn., 20, 475 (1984).

10. M. D. Gower and R. A. Shanks, Macromol. Chem. Phys., 206, 1015 (2005).

11. M. Van Gurp and J. Palmen, Rheol. Bull., 67, 5 (1998).

12. K. Sato and A. Toda, J. Phys. Soc. Jpn., 73, 2135 (2004).

13. D. H. Kaelble, J. Adhes., 1, 102 (1969).

14. J. P. Baetzold and J. T. Koberstein, Macromolecules, 34, 8986 (2001).

15. P. S. Gill, S. R. Sauerbrunn, and M. Reading, J. Therm. Anal., 40, 931 (1993).

16. M. Reading, D. Elliott, and V. L. Hill, J. Therm. Anal., 40, 949 (1993).

17. B. Wunderlich, Y. Jin, and A. Boller, Thermochim. Acta, 238, 277 (1994).

18. J. E. K. Schawe, Thermochim. Acta, 261, 183 (1995).

19. J. A. Forrest and R. A. L. Jones, "Polymer Surfaces, Interfaces and Thin Films," A. Karim \& S. Kumar, Ed., World Scientific, Singapore, 2000, P. 251.

20. P. S. Tucker, J. W. Barlow, and D. R. Paul, Macromolecules, 21, 1678 (1988).

21. K. Kimishima and T. Hashimoto, Macromolecules, 28, 3842 (1995). 